\title{
Blade Detection Robot
}

\author{
Nanyu Kuang* \\ North China Electric Power University(Baoding 071000), Hebei, China \\ 531157260@qq.com \\ * the corresponding author
}

Keyword: Centrifugal fan; Internet plus; Big data;Crawl along the surface

\begin{abstract}
At present, the traditional testing of wind farms is manual testing. But the safety and economy of manual detection are limited. Some companies use the UAV to detect fan blades failure. However, unmanned aerial vehicles equipped with infrared devices in the long-range detection is easily affected by the environment, the size of the type of injury is not easy to accurately determine the size. Therefore, based on the detection of various problems in the process of blades, we propose a robot to detect fan blades. The blade detection robot can generate pressure on the surface of the fan blade by centrifugal fan. This provides the necessary friction for the machine to crawl along the surface of the fan. The machine takes pictures of the fan blades by means of the high definition camera. In addition, the robot installed an infrared sensor to detect the surface of the fan blade. Data collected by robots and HD images are continuously analyzed. The use of big data and Internet plus concept, testing personnel will detect the information of the robot from discrimination, greatly improve the safety and reliability of wind turbine blade detection, also improves the detection efficiency.
\end{abstract}

\section{Research Background}

In the 21 st century, as the new energy promotes in our society, wind power has developed rapidly. But the wind is faced with sorts of cases, to name only a few, the maintenance of the fan blade is barely of the key issues.

At present large-scale wind turbine blades are shell structures which are made of glass fiber reinforced material. The hub height is about from 70 to 80 meters. Impeller diameter is from 70 to 90 meters. Leaf length is from 35 to 45 meters. The design life of the blade is 20 years. Maintenance items include: regular maintenance, which includes inspection, cleaning, adjustment, etc.; regular maintenance, with more comprehensive maintenance, focused inspection, cleaning, testing, measurement, inspection, oil lubrication and repair, parts to be replaced regularly; special maintenance; system equipment structure with a major transform in maintenance.

Fans are generally mounted in installation in sparsely populated areas, which brings great inconvenience in maintenance. The traditional manual detection of wind farm relies mainly on manpower testing, and quite a few companies use unmanned aerial vehicles to detect leaves. Nevertheless, the unmanned aircraft equipped with infrared devices is affected by the environment during detecting leaves in long distance. Additionally, the staff can not accurately determine the type and size of fan blades.

As a consequence, we put forward a kind of fan blade detection robot. Our robot service is based on regular maintenance. Maintenance period selects low wind or no wind days.

Current Status of Domestic Research.In the past few years, Hong Kong City University Intelligent Design, Manufacturing and Control Center (CIDAM) with the mainland universities, were committed to developing wall-wall-climbing robot, and they began to develop a Cartesian coordinate wall wall-climbing robot, which was composed of a translating mechanism and an adsorption device. By alternately moving the $\mathrm{X}$-axis and the $\mathrm{Y}$-axis, the corresponding sucker $\mathrm{Zx}$ and $\mathrm{Zy}$ are alternately adsorbed on the two axes to realize the movement of the robot. Since the robot had good stability, its movement was still not continuous because of the limited agency. In order to improve the efficiency, inspired by the tank crawler crawl way, CIDAM developed a 
continuous crawling of the multi-sucker single-chain climbing wall robot Cleanbot-IV. The advantage of the robot is that it can cross certain obstacles and be able to crawl continuously.

In 2010, Guangdong Thermal Power Corporation applied for a patented wind turbine automatic climbing machine. The most ingenious design of the climbing device is its stent: the bracket device has an upper electromagnet gripping mechanism and a lower [3] electromagnet gripping mechanism. The two electromagnets gripping mechanisms rely on the magnetic force and the working surface to freely switch the state of adsorption and release. A telescopic mechanism is provided between the upper electromagnet gripping mechanism and the lower electromagnet gripping mechanism to connect the two. The climbing machine utilizes the iron wall of the tower to hold and climb. Besides, it can be used to climb other iron walls. The structure of the climber consists of a ring-shaped main frame. As shown in Fig. 1

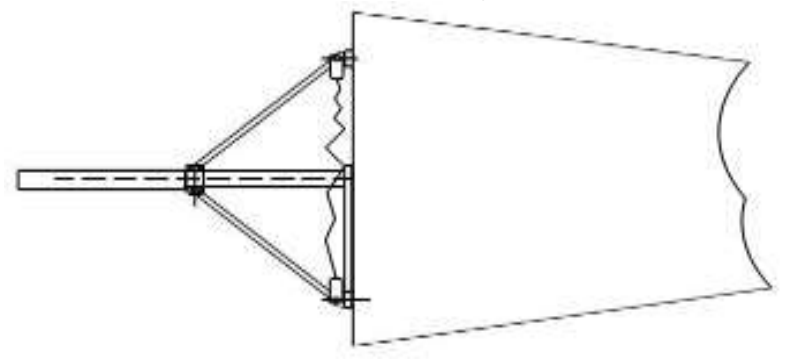

Figure 1. Climbing mechanism diagram

Surface inspection and cleaning work for high-rise buildings or marvelous structures are necessary for service life and safety precaution. Over the past 20 years, researchers have developed various types of wall-climbing robots, hoping to free people from dangerous and boring work. Thus, wall-climbing robots are generally divided into three categories: vacuum adsorption type, magnetic adsorption type and thrust type. At present, the most widely used is the vacuum adsorption type wall-climbing robot; this type of robot is divided into single sucker wall-climbing robot and multi-sucker robot. The former, such as Professor Hagong Wang Yan, presided over the development of the cleaning robot; the latter such as ROBINSPEC, Robug-II, Cleanbot-I and so on.

Status of Foreign Research The Korea Institute of Atomic Energy has developed a series of wall-climbing robots for the detection of various types of nuke industry equipment. There are wheeled magnetic adsorption type wall-climbing robots for detecting the leakage of pressurized water cooling reactor nozzles. As shown in Fig. 2. The robot is provided with a plurality of cameras.

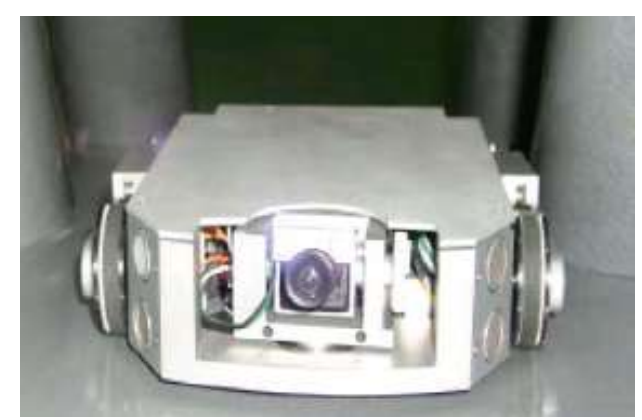

Figure 1. Wheeled magnetic adsorption type wall wall-climbing robot

GE has developed a robot that can climb quickly along wind turbine blade towers, as shown in figure 3. The robot uses a centrifugal fan to generate negative pressure, so it can be stably adsorbed on the outer surface of the tower, and the crawler-style movement will achieve rapid movement in the surface. The robot body can carry a camera or microwave scanner to observe the local area of the blade 

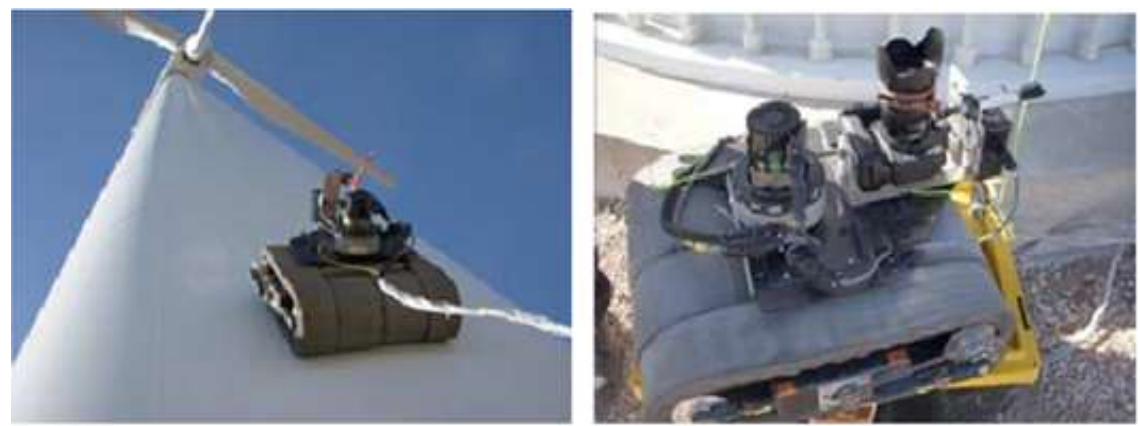

Figure 2. Fast wall-climbing robot

Schroeder Industrial Monitoring and Control Equipment Company developed wind turbine blade endoscopic detection robot SINGA100C. As shown in figure 4, the maximum cross-sectional diameter of the robot is $115 \mathrm{~mm}$. It can carry high-definition zoom camera and auxiliary light source and pour into the blade interior space, which can minimize the blind detection of manual detection.

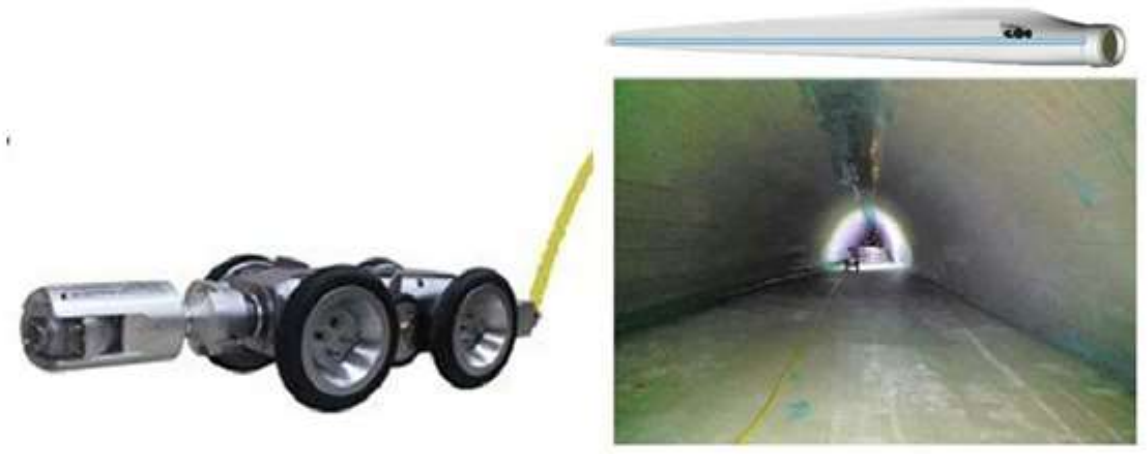

Figure 3. Wind power blade endoscopy detection robot

\section{Our Creativity on the Blade Detection Robot}

The blade detection robot can make pressure between itself and the blade surface of fans by its own centrifugal fan, and provide the necessary friction to crawl along the fan surface. Infrared sensor on the blade surface of fan makes non-destructive test. The data and the high-definition images collected by the robot can be analyzed continuously. Afterward the robot will automatically determine the detection information, which improves the safety, detection efficiency and reliability of blade detection of fans. As a consequence, the fan blade detection robot will transform the traditional operation and maintenance model from Fire-fighting operations to predictive operation. Meanwhile, it can carry out unit forecasting and physical examination and have an advance prevention to reduce losses.

It takes about one day to manually detect a fan and the cost is about $3000 \sim 4000$ yuan. The robot detects the blades at a speed of 8 meter per second time, which could accomplish detecting a fan blade; nevertheless, the cost is singly 1500 yuan. In comparison, detection cost of single fan sets aside next to $50 \%$ and it reduces fan downtime by $50 \%$. Thus it can be seen that the detection of fan blades of the robot is a product which has both market demand and market capacity

\section{Competition and Market Structure}

At this present stage, China's relevant robot is still in the stage of scientific research. Solely foreign imported robots can be used on the market. Foreign imported robots have these disadvantages: fancy price, corner on the market, patent blockade and difficulty in maintenance. Compared to the 
same series of foreign products, our robot has a more effective cost, lower price, better service and a better sale channel. At the same time, it will rely on humanity service to occupy the domestic market, and ultimately involves in the international market.

\section{Development Strategy}

Our company relies on the strong scientific and technological strength and perfect management of North China Electric Power University. We are composed of four highly educated college Students with different professional knowledge and wealthy experience. We plan to develop and launch wall-climbing robots currently. Our company is a modern technology enterprise which consists of development, management, and service.

Table 1 our action plan

\begin{tabular}{ccc}
\hline Starting time & Ending time & Task \\
\hline 20170102 & 20170228 & $\begin{array}{c}\text { Complete the 3D model of the robot } \\
\text { Draw and purchase parts group ,Assemble the robot }\end{array}$ \\
20170301 & 20170430 & $\begin{array}{c}\text { Complete the control system software and hardware design, the design of } \\
\text { the mechanical part of the rudder robot }\end{array}$ \\
20170501 & 20170527 & Debug the robot \\
20170528 & 20170830 & Complete the pilot experiment \\
\hline
\end{tabular}

\section{Target Positioning}

In the "fast fish eat slow fish" speed competition era, the success or failure of the blade detection robot's marketing depends largely on the ability to quickly win the market:

Attach importance to key areas: Hebei, Inner Mongolia Autonomous Region, Xinjiang, Jilin and other cities gathered wind power.

Emphasize key objects: each National wind power plants and industrial plants requiring difficult maintenance

Table 2 development plan

\begin{tabular}{|c|c|c|c|c|}
\hline Stage & Start stage & Rising stage & Mature stage & Long Term Goal \\
\hline objective & $\begin{array}{c}\text { fan blade } \\
\text { detection robot } \\
\text { Stable operation }\end{array}$ & $\begin{array}{l}\text { Accelerate the strategic } \\
\text { expansion, enhance the fan } \\
\text { blade detection robot } \\
\text { awareness, capital } \\
\text { accumulation to increase } \\
\text { turnover }\end{array}$ & $\begin{array}{l}\text { Stable } \\
\text { strategy to } \\
\text { improve } \\
\text { profitability }\end{array}$ & $\begin{array}{l}\text { Dig more target } \\
\text { customers and } \\
\text { fully cover the } \\
\text { potential market }\end{array}$ \\
\hline $\begin{array}{l}\text { Focal } \\
\text { point }\end{array}$ & $\begin{array}{l}\text { Optimize the } \\
\text { blade detection } \\
\text { robot and start the } \\
\text { marketing strategy }\end{array}$ & $\begin{array}{l}\text { Optimize maintenance, increase } \\
\text { publicity and expand your } \\
\text { influence }\end{array}$ & $\begin{array}{l}\text { Multi-level } \\
\text { marketing } \\
\text { strategy } \\
\text { adjustment }\end{array}$ & $\begin{array}{l}\text { Gradually } \\
\text { occupy a stable } \\
\text { market share }\end{array}$ \\
\hline
\end{tabular}




\section{Method of Sales}

Direct sales: in allusion to the national wind power enterprises, weuse the tool of geographic market segments and accomplish marketing to achieve three-dimensional sales.

Sales agent: we regard the blade detection robot dealers throughout the country as the target market, and develop a reasonable channel management mechanism to form a set of channel management system.

Franchise: we use the advantages of the product to form a complete product marketing system, and use the product platform to set up a franchise, unified image, brand and sales model to establish rationalization of the franchise mechanism and to improve the regulatory functions.

\section{Capital Structure and Scale}

The company mainly utilizes the team injection and other ways to finance:

We plan to invest 1 million yuan at the beginning. The source of funds includes the self-financing 600,000 yuan, short-term loans of bank loans 200,000 yuan, and the rate of bank short - term loans is $4.7 \%$. The total registered capital is 1 million.

\section{Risk Profile}

The company's products are the detection of the blade of the robot. We will not only have a wide market, but also be faced with an arm of risk.

This chapter evaluates the 10 possible risks in the future in macro environment, industry environment and the company.

The 10 potential risks are: natural risk, humanistic risk, economic risk, country policy risk, technology risk, cost risk, sales risk, market development risk, finance risk and management risk.

\section{References}

[1] LuoHaohua. Path planning and tactile compliance control of climbing wall robot for blade detection [D]. China Institute of Metrology, 2014.

[2] Zhao Zhi, Peng Guangjun. X-ray detection and microcomputer evaluation of cast steel fan blades [J]. Journal of Wuhan Automotive Industry University, 2000, (06): 72-75. [2017-10-03].

[3] ZHANG Zhi-feng, YAO En-tao, SHI Yu. Endpoint Detection Algorithm for Sound Signal Based on Wavelet Analysis and MFCC [J]. Electronic Measurement Technology, 2016, (07): 62-66.

[4] Du Yongfeng, Hou Bin, Li Wanrun, Liu Peng. Lamb wave detection method and its application in fan blade structure health monitoring [J]. Non-destructive testing, 2015,37 (09): 80-86. [2017- 10-03].

[5] Huidong Chang. Based on the main passive technology integration of fan blade structure health monitoring technology research [D]. Nanjing University of Posts and Telecommunications, 2016. 